\title{
High-throughput widefield fluorescence imaging of 3D samples using deep learning for 2D projection image restoration
}

Edvin Forsgren ${ }^{1{ }^{*}}$, Christoffer Edlund ${ }^{2 \diamond}$, Miniver Oliver ${ }^{3 \propto}$, Kalpana Barnes ${ }^{3}$, Rickard Sjögren ${ }^{2}$, Timothy R. Jackson ${ }^{3}$

1 Computational Life Science Cluster (CLiC), Department of Chemistry, Umeå University, Linnaeus väg 10, 90736 Umeå, Sweden

2 Sartorius Corporate Research, Sartorius Stedim Data Analytics AB, Östra

Strandgatan 24, 90333 Umeå, Sweden

3 Sartorius BioAnalytics, Essen BioScience, Ltd., Units 2 \& 3 The Quadrant, Newark

Close, Royston, Hertfordshire, SG8 5HL, United Kingdom

OThese authors contributed equally to this work.

oCurrent Affiliation: Biopharm Discovery, GlaxoSmithKline, Gunnels Wood Rd, Stevenage, Hertfordshire, SG1 2NY, United Kingdom

IMembership list can be found in the Acknowledgments section.

* edvin.forsgren@umu.se

\section{Abstract}

Fluorescence microscopy has become a core tool for visualizing and quantifying the spatial and temporal dynamics of complex biological processes. Thanks to its low cost and ease-of-use, widefield fluorescent imaging remains one of the most widely used fluorescence microscopy imaging modalities. To accomplish imaging of 3D samples, conventional fluorescence imaging entails acquiring a sequence of $2 \mathrm{D}$ images spaced along the z-dimension, typically called a z-stack. Oftentimes, the next step is to project the $3 \mathrm{D}$ volume into a single $2 \mathrm{D}$ image, as $3 \mathrm{D}$ image data can be cumbersome to manage and challenging to analyze and interpret, effectively limiting the utlity of z-dimensional information. Furthermore, z-stack acquisition is often time-consuming and consequently 
may induce photodamage to the biological sample, which are both major hurdles for its application in experiments that require high-throughput, such as drug screening. As an alternative to z-stacks, axial sweep acquisition schemes have been proposed to circumvent these drawbacks and offers potential of 100-fold faster image acquisition for 3D-samples compared to z-stack acquisition but unfortunately results in blurry, low-quality raw $2 \mathrm{D}$ z-projected images. We propose a novel workflow to combine axial z-sweep acquisition with deep learning-based image restoration, ultimately enabling high-throughput and high-quality imaging of complex 3D-samples using 2D projection images. To demonstrate the capabilities of our proposed workflow, we apply it to live-cell imaging of 3D tumor spheroids and find we can produce high-fidelity images appropriate for quantitative analysis. Therefore, we conclude that combining axial z-sweep image acquisition with deep learning-based image restoration enables high-throughput and high-quality fluorescence imaging of complex 3D biological samples.

\section{Introduction}

Fluorescence microscopy has become an indispensable tool to study biological phenomena, enabling countless discoveries over the past several decades. For live-cell imaging in particular, fluorescence microscopy can enable interrogation of the spatial and temporal dynamics of complex biological processes. By using fluorescent proteins (FPs) to label endogenous cell signaling molecules, one can gain insight into the heterogeneity and kinetics of changes to cellular processes over time through fluorescence intensity measurements. However, fluorescence imaging has inherent challenges and requires careful balancing of light exposure to samples and overall acquisition time with the signal-to-noise ratio. [1,2]. Overexposure of samples can cause FP photobleaching or phototoxicity, while underexposure will generate noise-dominated images. Oftentimes, to obtain meaningful data, acquisition parameters must be uniquely tuned to the specific FP and biological sample.

These matters are further complicated when considering three-dimensional (3D) samples. For the most widely used modalities of fluorescent imaging, i.e. widefield and confocal, conventional 3D fluorescent imaging entails acquiring a step-wise sequence of 
two-dimensional (2D) optical slices spaced across the axial dimension, which is commonly referred to as a z-stack [2]. Optimal axial sampling rates are determined by the z-axis resolution of the microscope [3] but are usually impractical for large samples, which may require images at hundreds of z-locations. Additionally, once a z-stack is acquired, because many applications do not require axial information and 3D data becomes cumbersome to manage, much of the time it is sufficient to project 3D volumes into a single 2D image [4]. In fact, many software packages exist that enable this feature of producing z-projected images $[5,6]$.

Despite technical challenges, recent trends in cellular research continues to see focus shift from 2D monolayers of cells toward more physiologically relevant three-dimensional 3D models, including tumor spheroids and organoids $[7,8]$. 3D models facilitate greater insight into microenvironmental factors and spatial interactions, incorporating elements that are better representative of conditions seen in vivo. While approaches such as lattice light sheet fluorescence microscopy promise high-throughput, high-quality fluorescent imaging of such samples [9], they require sophisticated hardware and user expertise. To increase the throughput for widefield fluorescent imaging, an alternative acquisition scheme was proposed to couple axial sweeping with light modulation and point-spread function (PSF)-based image restoration to generate an axially compressed $2 \mathrm{D}$ image of a $3 \mathrm{D}$ sample [10]. While this strategy was promising in its 2 - to 10 -fold acquisition speed increase, its hardware requirements and complex traditional computer vision-based approach limits its practicality and implementation to complex biological samples. More recently, an acquisition scheme was proposed utilizing sweeping synchronized galvanometric mirrors in a multi-angle projection system to accelerate imaging speeds by a factor of $>100$ [4]. While indeed promising, this technique requires a specialized hardware setup and was only tested in confocal and light-sheet fluorescence microscopy, making its applicability unclear for widefield fluorescence imaging.

Whereas traditional PSF-based image restoration is incredibly challenging for complex 3D samples, convolutional neural networks (CNNs) are now routinely used for such difficult computer vision problems. Across various 3D fluorescence imaging studies, CNNs are used to segment nuclei $[11,12]$ and cell bodies $[13,14]$, image restoration $[15,16]$, image super-resolution [17], virtual refocusing [18,19], optimization of phase mask filters for PSF-engineering [20,21] as well as blind deconvolution in 2D 
widefield fluorescence imaging [22,23]. Additionally, recurrent neural networks (RNNs)

were recently used to reconstruct a complete $3 \mathrm{D}$ volume with far fewer optical sections

than typically needed [24]. However, while CNN-based machine learning provide a

flexible framework for many computer vision problems, they have not yet been used to enable high-throughput fluorescent imaging of complex 3D samples without acquiring multiple z-slices of samples, ultimately limiting throughput.

In this paper we present a novel workflow to generate high-quality $2 \mathrm{D}$ projection images of entire 3D fluorescent biological specimens while minimizing exposure times and maximizing throughput (summarized in Fig. 1). Using live 3D tumor spheroids, we collected paired datasets comprising conventional projected z-stacks (Fig. 1a) and the corresponding images formed from a fast axial sweep acquisition, which we are terming a z-sweep image (Fig. 1b). We then train CNN-based conditional generative adversarial neural networks on the image pairs, in order to achieve a model that can faithfully reconstruct high-quality $2 \mathrm{D}$ projections of z-stacks from the comparatively low-quality z-sweep images (Fig. 1c). By combining state-of-the-art deep learning-based computer vision with high-throughput image acquisition, we enable low-phototoxicity, real-time quantitative analysis of live 3D samples using widefield fluorescence imaging.

Fig 1. Schematic overview of the proposed workflow. Firstly, we collect a dataset of paired z-projection and z-sweep images. Z-projection images are acquired by first collection a stack of fluorescent images along the z-direction and projecting them down to a high quality 2D-image (A). On the other hand, z-sweeps are acquired by axial sweeping through the z-dimension yielding low quality images, albeit with very high throughput (B). We train a convolutional neural network model to predict the high-quality z-projection images from z-sweep images, which later enables us to bypass the need of a z-stack in order to acquire high-quality images with high throughput (C).

\section{Methodology}

Formally, we aim to map a z-sweep image, $S \in \mathbb{R}^{h \times w}$, to the corresponding z-projection image, $P \in \mathbb{R}^{h \times w}$, by means of a generator $G: \mathbb{R}^{h \times w} \rightarrow \mathbb{R}^{h \times w}$ such that $G(S)=\hat{P} \approx P$, where, $G$ is a CNN parameterized by $\theta$. Given a dataset of $N$ pairs of z-sweep and z-projection images, we train $G$ to minimize the loss function $L: \mathbb{R}^{h \times w} \times \mathbb{R}^{h \times w} \rightarrow \mathbb{R}$ to find optimal parameters: 


$$
\hat{\theta}=\underset{\theta}{\arg \min } \sum_{i=1}^{N} L\left(G\left(S_{i} \mid \theta\right), P_{i}\right) .
$$

The sections that follow will describe the CNN architectures investigated in this paper and the loss functions used to train them.

\section{Image Generator}

In this work, all generators are based on the CNN-based encoder-/decoder architecture

U-Net (Fig. 2a), which has seen frequent use in the field of image-to-image

transformation in medical imaging [25]. U-Net operates by encoding images into feature

maps, by concurrently reducing spatial resolution and increasing dimensionality, before

decoding those feature maps into an image of the desired resolution, using

skip-connections between encoder and decoder-blocks to preserve spatial information.

For each up- and downsampling operation, the standard U-Net block comprises a $3 \times 3$

convolution, batch normalization, and rectified linear unit (ReLU)-activation (Fig. 2b).

Fig 2. Diagrams of ANN architectures used in this study. (a) U-Net

architecture, the encoder/decoder architecture, which our image-to-image generator models are based on. Each upsampling uses bilinear interpolation to double image size and each downsampling uses max pooling to half image size. (b) The standard U-Net convolutional block, which contains a $3 \times 3$ convolution $\left(F_{3 \times 3}\right)$, batch normalization (BatchNorm) and standard ReLu. (c) PatchGAN, which comprise the discriminator of our CGAN-based models. The two channels in the first PatchGAN-layer indicate the input z-sweep image stacked onto the corresponding z-projection, either predicted or measured. Each arrow indicates a convolutional block containing a convolution with a $4 \times 4$ kernel and stride of 2 (Conv 4x4), batch normalization (BatchNorm) and leaky ReLU activation function with alpha=0.2 (LReLU). (d) OSA block as adopted from [26], which our improved U-Net variants use as replacement for the standard U-Net convolutional block. Here, $F_{3 \times 3}$ and $F_{1 \times 1}$ indicate $3 \times 3$ and $1 \times 1$ convolutional layers, $F_{\text {AdAvg }}$ adaptive average pooling over spatial dimensions, $\otimes$ indicate element-wise multiplication and $\oplus$ element-wise addition. $F_{A d A v g}$ followed by the $1 \times 1$ convolution and the hard Sigmoid activation make up the efficient Squeeze-and-Excitation (eSE)-module of the OSA-block. Note that $F_{1 \times 1}$ projects the concatenated tensor to the same dimensions as input tensor and that the element-wise multiplication is performed along the channel-axis and broadcasted over the spatial dimension.

To improve upon the standard U-Net, we investigated a version where the standard block is replaced by One-shot Aggregation (OSA)-blocks with efficient squeeze-and-excitation (eSE)-modules [26] (Fig. 2d). OSA-blocks were proposed to optimize use of skip connections by reducing feature redundancy, in order to ultimately 
improve predictive performance while still being computationally efficient. eSE-modules $\quad{ }_{87}$ are a development of squeeze-and-excitation (SE)-modules that were proposed to adaptively re-weight channels of encoded images in each convolutional block to further improve predictive performance [27].

To provide baseline generator models, we train both the standard U-Net and the OSA-U-Net to minimize the L1-reconstruction error of the predicted z-projection compared to the ground truth. Formally, the L1 reconstruction error is given by

That is, the L1-reconstruction error sums the absolute pixel-wise prediction errors.

\section{Conditional Generative Adversarial Network}

Whereas using purely reconstruction-based losses in an image-to-image application seems intuitive, in practice it often leads a network to favor proposals which are overly smooth or blurry. This is where a generative adversarial network (GAN)-based approach can offer improvement by applying two sub-models, a generator that proposes images and a discriminator to classify the proposal as real or fake, to drive proposals to become more realistic [28]. Based on the work of Isola et al., we investigated the use of conditional GANs (CGANs) to improve the generated z-projection images (summarized in Fig. 3). In the CGAN approach, we have our generator, $G$, which is trained to predict the image $G(S)=\hat{P}$ based on $S$, where we wish $\hat{P}$ to be indistinguishable from the the target $P$. We then add a secondary loss term to optimize $G$ to fool a discriminator model, $D$, which is trained to predict whether $P$ or $\hat{P}$, conditioned on the input $S$, is real or or not. That is, the discriminator is defined as $D: \mathbb{R}^{h \times w} \times \mathbb{R}^{h \times w} \rightarrow I^{\hat{h} \times \hat{w}}$, where $I=[0,1]$ indicates the closed interval of predicted probabilities that each output node corresponds to a real pair and $\hat{h}$ and $\hat{w}$ are the output dimensions used in the CGAN PatchGAN-framework. 
Fig 3. Schematic illustration of the conditional generative adversarial network (CGAN)-approach used to improve prediction of z-projection of images. $\bigoplus$ indicate stacking of the images. During training, the generator $G$ predicts a z-projection image $\hat{P}$ based on the input sweep image $S . S$ is stacked onto $\hat{P}$ to create a two-channel image, which the discriminator $D$ attempts to classify as fake $D^{f}$ (a). To provide negative examples, real z-projection images $P$ are similarly stacked with z-sweeps and the discriminator then attempts to classify them as real $D^{r}$ (b). The discriminator is optimized to accurately classify z-projection images as real or fake, while the generator network is optimized to make the discriminator prediction more difficult. The scale bar in the leftmost image applies to all images.

Formally, we optimize $G$ to minimize the weighted sum of the L1-loss (2) and an adversarial loss based on the discriminator prediction that is given by acersarial loss based on the disiminator prediction that is given by

$$
L_{G}=\lambda_{L 1} L_{L 1}(P, \hat{P})+\mathbb{E}\left[(D(S, \hat{P})-R)^{2}\right]
$$

where $\lambda_{L 1} \in \mathbb{R}$ is the weight of the L1-reconstruction loss, $R$ the $\hat{h} \times \hat{w}$ matrix of soft real labels, meaning that $R_{i j} \in[0.9,1]$ for each $i \in[1, \hat{h}]$ and $j \in[1, \hat{w}]$ to use so called one-sided label smoothing, which Salimans et al. found to improve GAN-training [29]. The discriminator then minimizes the loss given by

$$
L_{D}=\frac{1}{2} \mathbb{E}\left[(D(S, P)-R)^{2}+(D(S, \hat{P})-F)^{2}\right]
$$

where $F$ is the $\hat{h} \times \hat{w}$ matrix of fake labels, meaning that $F_{i j}=0$ for each $i \in[1, \hat{h}]$ and $j \in[1, \hat{w}]$.

The CGAN-discriminator uses the PatchGAN architecture (Fig. 2c), which has been found to improve texture quality of predictions [28]. Instead of the standard

GAN-discriminator which performs binary classification of the input image as either real or fake [30], the PatchGAN-discriminator can be viewed as a "sawed off" CNN, which outputs a 2D-tensor where the output neurons represent the discriminator classification of overlapping patches. To train the CGAN, the generator- and discriminator-weights are updated in an alternating fashion (illustrated in Fig. 3).

Discriminator weights are frozen during the first phase while the the generators weights are updated, optimizing the loss equation based on discriminator outputs (3). During the second phase, the generator weights are frozen to turn attention to the discriminator weights, which are updated to reduce loss defined in (4). 
Fig 4. Graphical summary of establishment and image acquisition of 3D tumour spheroid datasets. To establish multi-spheroids (a), MCF7-NR cells were seeded onto a layer of polymerized Matrigel and imaged in an Incucyte $\mathbb{R}$ over time. Brightfield, z-projection and z-sweep fluorescence images acquired 10 days post seeding are shown. In a single spheroid assay format (b), A549-NR cells were seeded into a ULA plate and allowed to form large single tumor spheroids and imaged as above. To visualize invasive cancer biology, MDA-MB-231-NR cells were embedded in $50 \%$ Matrigel and imaged daily 8 to 11 days post seeding (c).

\section{Experiments}

\section{Acquisition of datasets}

To establish proof of concept across multiple biological protocols, we tested our proposed workflow on three common protocols for culturing live 3D tumor spheroids, each of which present different imaging challenges. All cell lines were stably expressing a nuclear-restricted mKate2 FP following transduction with the Incucyte@ NucLight Red lentivirus reagent (Sartorius). Because the FP is nuclear restricted, it can be used to measure cell viability, i.e. a loss of viability results in a compromised nuclear membrane to cause a loss of fluorescence. The sections below describe these three protocols.

\section{Single-spheroid preparation}

In this protocol, cancer cells are encouraged to form a single large tumor spheroid (Fig. ${ }^{140}$

$4 \mathrm{a})$, the size of which can present unique imaging challenges including limited light penetration and excess light scattering [31]. Four common immortalized cancer cell lines were used: A549 (lung cancer), MCF-7 (breast cancer), MDA-MB-231 (breast cancer) and SK-OV-3 (ovarian cancer). Cells were seeded into an ultra-low attachment round-bottom 96-well plate (Corning \#7007), gently centrifuged at 150x g for 10min, and allowed to naturally form a single large spheroid of up to approximately $1 \mathrm{~mm}$ in diameter over a 10 day period . At 3 days post seeding, $1 \mu \mathrm{M}$ of the cytotoxic compound camptothecin (CMP) was added to half of the wells. Spheroids continued to be monitored at regular intervals by the standard Incucyte@ Spheroid module, before the custom fluorescence image acquisition was performed at 10 days post cell seeding once the control tumor spheroids had reached a size of approximately $1 \mathrm{~mm}$ in diameter. 


\section{Single-plane multi-spheroid preparation}

In this approach, rather than forming a single large spheroid, cancer cells are seeded on $\quad{ }_{153}$ top of a flat extracellular matrix base to facilitate multiple tumor spheroid growth.

Furthermore, tumor spheroids grow in what is effectively a single optical plane, simplifying matters for imaging (Fig. 4b). To prepare for imaging, a flat-bottom 96-well plate (Corning \#3595) was coated with $40 \mu \mathrm{L}$ of $4.5 \mathrm{mg} / \mathrm{mL}$ Matrigel $\AA$ and polymerized at $37^{\circ} \mathrm{C}$ for $30 \mathrm{~min}$. A549 or MCF-7 Cells were then seeded on top of polymerized matrix and allowed to form spheroids. Multi spheroid formation was monitored by regular brightfield imaging with the standard Incucyte@ Spheroid module, before the customized z-sweep and z-stack fluorescence image acquisition workflow was carried out at 10 days post cell seeding. Brightfield images were also collected in order to visualize the tumor spheroids.

154

155 156 157

\section{Embedded multi-spheroid preparation}

To visualize invasive cancer biology, an embedded multi-spheroid assay was prepared, which is the most complicated from an imaging perspective. Cancer cells are embedded within a volume of extracellular matrix, pushing the limits of the acquisition to resolve spheroids residing at varying z-locations (Fig. 4c). Here, either MCF-7 or MDA-MB-231 cells were directly suspended in $50 \mu \mathrm{L}$ of $50 \%$ Matrigelß. The suspension was placed in each well of a cold 96-well flat bottom plate (Corning \#3595), gently distributed across the entire bottom of the well and allowed to polymerize at $37^{\circ} \mathrm{C}$ for $20 \mathrm{~min}$ resulting in a column of Matrigel that is approximately $1.5 \mathrm{~mm}$ tall. At 7 days post seeding, $1 \mu \mathrm{M}$ CMP was added to half of the wells. In order to obtain kinetic data with which to validate our acquisition strategy, we performed the custom fluorescence image acquisition at 8, 9, 10 and 11 days post seeding.

\section{Image acquisition}

Imaging was performed using customized acquisition software written to operate the motorized widefield fluorescence microscope within the Incucyte@ S3 Live-cell imaging system (Sartorius). All images were collected using a 4X magnification objective (Navitar, NA=0.2, FN=26.5), CMOS camera (Basler Ace 1920-155um) and the Incucyte 
Red/Green optical module (Sartorius). While these experiments were performed on an ${ }_{181}$ Incucyte, where the camera moves in relation to a stationary sample, the acquisition $\quad{ }_{182}$ strategy could presumably apply to any widefield fluorescent microscope with a motorized stage. For all experiments, the camera was moved to the center of the sample microwell and proceeded visualize fluorescence in the tumor spheroid using two image acquisition paradigms.

In the first paradigm, the traditional z-stack strategy was carried out. Here, a volume of either $750 \mu \mathrm{m}$ (single spheroid or single-plane multi-spheroid) or $1.5 \mathrm{~mm}$ (embedded multi-spheroid) was collected as a step-wise image series, where the camera repeats a plane-by-plane sequence of moving upward $25 \mu \mathrm{m}$ along the z-axis starting from the bottom of the microwell, at each location pausing to acquire an image with a $50 \mathrm{~ms}$ exposure time. Because the optimal Nyquist sampling rate is impractical and results in substantial photobleaching, the z-step distance was instead selected to be within the depth-of-field of the objective $(\mathrm{DOF}=25.3 \mu \mathrm{m})$ in order to balance sampling rate and axial resolution. To minimize shot noise, we took 3 consecutive frames and averaged them together before moving to the next z-location. This sequence is then repeated throughout the entire volume. Each image series is then projected (Fig. 1a, left) using either a standard maximum intensity z-projection (see Supplemental Materials) or a custom z-projection algorithm [32]. This custom projection scheme uses a neighborhood-based texture filter to determine the location in the z-stack in a manner similar to other texture-based projection algorithms [33], resulting in an image where the surface of each tumor spheroid object is in focus.

In the second acquisition paradigm, the camera covers the same axial distance as before by moving continuously along the z-axis at a speed of $1.25 \mathrm{~mm} / \mathrm{s}$, with the goal of producing a single image where intensity is integrated across the z-dimension. While a single long exposure would have been preferred to accomplish this task, it was not possible to accomplish without saturating the 12-bit camera. Instead, the camera moved continuously while a consecutive series of $50 \mathrm{~ms}$ exposures with each exposure translating to motion across an approximately $65 \mu \mathrm{m}$ distance. Consecutive exposures were summed into a single image to obtain the final z-sweep image (Fig. 1a, right).

Both z-stack projections images and summed z-sweeps were normalized by their effective exposure time prior to neural network training, which was critical for 
maintaining the correlation of intensity values between the paired data to enable accurate neural network training. Care was also taken to minimize time between image ${ }^{214}$ acquisition paradigms for a given sample to maintain optimal data pairing for neural network training. In all cases, accompanying transmitted light bright-field image z-stacks were also collected in order to visualize the tumor spheroids.

\section{Data and training specifics}

The single plane multi-spheroid dataset was used to train all models and consists of 96 219 different pairs of z-sweep and z-projection images. Out of these 96 images, 86 of them 220 were chosen to be used for model training and the 10 remaining images were used to ${ }_{221}$ validate the models. Data augmentation was used to enlarge the dataset, meaning that ${ }_{222}$ each pair of $1536 \times 1152$ images was cropped into multiple matching $256 \times 256$ sub-images at random locations. Cropped images were also flipped horizontally and vertically at random with a probability of $25 \%$ for both cases. Using data augmentation, ${ }^{225}$ the final training set consisted of 344 images and the validation set 40 images. All 226 images were log-transformed during training. In comparison to batch-normalization, ${ }_{227}$ log-transformation keeps the relation of intensity between all the images the same and the network will therefore be able to learn to predict fluorescent intensity in a dynamic range.

The generator models follow the U-Net and OSA U-Net architectures described in section Image Generator. The CGAN-discriminator follows a PatchGAN-architecture, $\quad 232$ which consists of a CNN with basic convolutional blocks, batch normalization and leaky ReLu activations with alpha $=0.2$, outputting 2 D-tensors corresponding to $70 \times 70$ pixel overlapping receptive fields (see Fig. 2c). To train the models, we used a mini-batch stochastic gradient decent configured with an Adam-optimizer [34], a learning rate of 0.0002 and batch size of 2. To train the CGAN-based models, the weight for the generator L1-loss, $\lambda_{L 1}$, was set to 10 . Also, one-sided label smoothing [29] was used for real images, meaning that a tensor of random values in the interval $[0,0.1]$ was subtracted from their target labels. Model check-pointing was used, meaning that all networks were trained for a maximal number of 1250 epochs and the models corresponding to the minimum validation error were used for further 
Table 1. Z-projection reconstruction performance on test set images as quantified by Frechet Inception Distance (FID), Peak Signal to Noise Ratio (PSNR) and Structural Similarity (SSIM). Performance is reported for the compared models as well as the input z-sweep to provide a baseline. Arrows indicate direction of improvement, meaning that an upward arrow indicates that a larger score is better and the opposite for a downward arrow.

\begin{tabular}{|l|c|c|c||c|c|c|}
\hline & \multicolumn{3}{|c||}{ Single-spheroid } & \multicolumn{3}{c|}{ Multi-spheroid } \\
\hline & FID $(\downarrow)$ & PSNR $(\uparrow)$ & SSIM $(\uparrow)$ & FID $(\downarrow)$ & PSNR $(\uparrow)$ & SSIM $(\uparrow)$ \\
\hline Z-sweep & 312.73 & $16.11 \pm 4.75 \mathrm{~dB}$ & $0.9963 \pm 0.0031$ & 294.25 & $17.97 \pm 4.05 \mathrm{~dB}$ & $0.075 \pm 0.045$ \\
\hline OSA-CGAN & 147.16 & $11.90 \pm 8.69 \mathrm{~dB}$ & $0.9947 \pm 0.0057$ & 99.07 & $23.66 \pm 4.8 \mathrm{~dB}$ & $0.107 \pm 0.041$ \\
\hline CGAN & 187.39 & $10.51 \pm 9.48 \mathrm{~dB}$ & $0.9890 \pm 0.0152$ & 121.47 & $20.44 \pm 5.74 \mathrm{~dB}$ & $0.089 \pm 0.037$ \\
\hline OSA-U-Net & 181.76 & $14.09 \pm 9.50 \mathrm{~dB}$ & $0.9953 \pm 0.0059$ & 113.59 & $22.45 \pm 6.39 \mathrm{~dB}$ & $0.109 \pm 0.041$ \\
\hline U-Net & 196.24 & $11.07 \pm 9.43 \mathrm{~dB}$ & $0.9902 \pm 0.0135$ & 105.47 & $21.02 \pm 5.64 \mathrm{~dB}$ & $0.081 \pm 0.035$ \\
\hline
\end{tabular}

experiments. Specifically, U-Net was trained for 1027 epochs, OSA U-Net for 1014, ${ }_{243}$ CGAN for 1217 and OSA-CGAN for 1227. For the CGAN-models, the validation error ${ }^{244}$ was given by the supervised L1-loss. The code can be found on GitHub [35].

\section{Results}

\section{Model evaluation}

To determine what type of CNN or method is most suitable for z-projection image reconstruction from z-sweep images, we compared four different models trained on the single-plane multi-spheroid dataset. All image generator models are based on U-Net [25], which is modified by adding adversarial training (CGAN) and/or replacing the standard convolutional blocks with OSA-blocks. To evaluate which model is able to predict the most accurate z-projection images from z-sweep images, we compare the image reconstruction performance using Frechet Inception Distance (FID) [36], Peak Signal to Noise Ratio (PSNR) and Structural Similarity (SSIM) on single-spheroid and single-plane multi-spheroid image pairs not used during training (see Fig 4.a, Fig 4.c, and Table 1). FID, PSNR and SSIM all evaluate the perceived quality of the predicted image compared to a reference. While PSNR and SSIM are widely used in classical signal processing, FID was developed specifically to evaluate the quality of natural images generated by generative adversarial networks.

Out of the investigated models, OSA-CGAN performs best overall (Table 1). OSA-CGAN has the lowest FID (where lower is better) on both the multi- and 
single-spheroid datasets. In terms of PSNR and SSIM, while the results are not as conclusive, OSA-CGAN is among the best ranking models for both metrics and datasets. $\quad 264$ Indeed, we find that OSA-CGAN produces the images that are qualitatively the most 265 similar to the z-projection ones (see Fig. 5). We also find that both investigated modifications to U-Net, OSA-blocks and CGAN, contribute to the improved performance. In terms of FID on the single-spheroid data, both modifications improve relative to $\mathrm{U}-\mathrm{Net}(\mathrm{FID}=181.76$ for OSA-blocks and 187.39 for CGAN compared to 196.24 for U-Net) but less than them combined $(\mathrm{FID}=147.16)$. While on the multi-spheroid images, each modification degrade performance (FID = 113.59 for OSA-blocks and 121.47 for CGAN compared to 105.47 for U-Net) but improve performance when combined $($ FID $=99.07)$. We draw similar conclusions from PSNR and SSIM. Qualitatively, we find that both CGAN and OSA-blocks improve the sharpness of the generated images, however not over the full dynamic range of the images and appear comparatively blurrier to the z-projection. Our proposed OSA-CGAN on the other hand, generates perceptually sharp images over the full dynamic range (Fig. 5 and 6$)$.

Fig 5. Illustrative examples of z-sweeps, z-projections and model predicted reconstructions for the compared models. The scale bar in the bottom left image is $50 \mu \mathrm{m}$ and applies to all images. The brightness of the images are relative for visual purposes.

Fig 6. Qualitative reconstruction performance of OSA-CGAN. On single-spheroid images (a) and multi-spheroid ones (b). The brightness of the images are relative for visual purposes.

\section{Fluorescence fidelity}

A common use case of 3D fluorescent imaging of tumor spheroids is to quantify the mean fluorescence intensity within the spheroid to measure biological response, e.g., cell health and viability. In order to use predicted z-projections in such applications, it is critical to not only predict images that are perceptually similar, but they also need to carry the same information without introducing systematic bias. To assess whether our proposed OSA-CGAN allows for the same spheroid-wise quantification as the z-projection, we detect individual spheroids in the multi-spheroid dataset and quantify 
the spheroid-wise logged sum of fluorescence intensities and compare to

z-projection-based quantification (Fig. 7).

Fig 7. Localization and quantification log-sum fluorescence intensity of tumor spheroids in the multi-spheroid dataset. Based on the z-projection (a) and the OSA-CGAN predictions (b) compared in a scatter plot (c) alongside the quantification based on the z-sweep images.

We find that OSA-CGAN allows for equivalent quantification as the z-projection without introducing systematic bias $\left(R^{2}=99.4 \%\right.$, slope $=1$, Fig. 7c). In comparison, ${ }_{290}$ the z-sweep intensities are noisier compared to the predicted z-projections and systematically underestimates the intensity. We conclude that OSA-CGAN faithfully reproduces the z-projection fluorescence intensities on an individual tumor spheroid basis.

Fluorescence-based measure of cell death in embedded spheroids

Fig 8. Time courses of nuclear-restricted mKate2 fluorescence intensities of embedded tumor spheroids. Quantified based on z-sweeps (a), OSA-CGAN predictions (b), and z-projections (c). Note the different scale of y-axis in A.

To demonstrate that our workflow allows equivalent interpretation in biologically relevant datasets as traditional fluorescence microscopy, we investigated fluorescence intensity-based measurements from the different acquisition paradigms to quantify loss of cell viability over time. Taking our best performing model from above, we applied the OSA-CGAN to z-sweeps of a dataset comprising embedded MDA-MB-237 and MCF-7 tumor spheroids, which were either vehicle or CMP-treated to induce cell death. Here, because tumor spheroids are embedded throughout the Matrigel@, spheroids reside at different locations along the z-dimension. To quantify fluorescence, we segmented the bright-field images of the tumor spheroids using a custom version of the Incucyte@ Spheroid Analysis module. Using those bright-field segmentation masks, we quantified the average mean fluorescence intensity of tumor spheroids in each image to compare the raw z-sweep, OSA-CGAN prediction and z-projection images. Masked objects with area less than $1200 \mu \mathrm{m}^{2}$ were not included in the analysis, in order to exclude debris or other bright-field imaging artifacts. Results are shown in Fig. 8. 
Using this strategy, we find that the predictions from our proposed method correspond very well to the gold standard z-projection (Fig. 8b compared to c). Both display similar trends over time, both in terms of absolute intensities and relative changes, to show the loss in cell viability in response to CMP treatment compared to the control. In comparison, the quantification based on the raw z-sweeps are underestimated relative to the z-projection (Fig. 8a). Although the general trends are similar, the z-sweep quantification shows that early time-point intensities of treated MDA-MB-237 are higher in terms of absolute intensities. For example, at the first time point, the CMP-treated MDA-MB-237 exhibit higher intensity than the vehicle-treated MCF-7 at the first time point and is higher than both MCF-7 cultures at the second time point. This relationship is not observed in the quantification based on z-projection images nor predicted images. This suggests that there is potential for mischaracterizing fluorescence-based data of the raw z-sweep acquisition, which can actually be corrected by our trained OSA-CGAN model.

\section{Discussion}

Widefield fluorescence imaging has been an indispensable tool for advancement of biological research for decades. However, experienced users are all too familiar with the trade-offs that need to be considered when imaging a 3D sample, namely balancing throughput and minimal risk of phototoxicity and photobleaching with maximal signal-to-noise and axial resolution [2]. Here, we present an approach to circumvent those trade-offs without the need of additional hardware, by coupling deep learning-based image restoration with an unconventional acquisition paradigm to achieve high-quality, quantitative $2 \mathrm{D}$ projection images of $3 \mathrm{D}$ samples. This proposed method enables high-throughput widefield fluorescence imaging in a manner that has not previously been feasible for 3D samples, permitting lines of investigation that have previously only been possible in 2D models or with specialized optical setups. For example, efficient anti-cancer compound screening, where fluorescence data is typically used to quantify cell health and death, requires many different drugs and concentrations to be tested simultaneously. Because such high-throughput is a requirement for these experiments, researchers either use 2D cell culture models, which are not necessarily 
relevant to true in vivo conditions, or they perform very limited axial sampling of their $\quad 340$ 3D samples, which risks biasing or leaving behind data. Not only does the proposed 341 method capture fluorescent images in a fast, high-throughput manner to generate qualitatively impressive images (Fig. 6), but it also maintains the fidelity of quantitative measurements (Fig. 7) making it suitable for measuring biological response, e.g., cell death in response to drug treatment (Fig. 8). This demonstrates its utility for quantitative fluorescent imaging of 3D samples, which enables the same data measurements in a fraction of the time of the conventional z-stack approach.

Worth noting is that the z-stack approach suffers from halo-like artefacts surrounding the cell clusters, due to light scattering typical to widefield fluorescence [3]. This artefact is apparent with close inspection of the ground truth images in Fig. 6. Because these artefacts are present in the training dataset, one could expect these to negatively affect the model prediction; however, this is not the case. Instead, the model learns to ignore these in favor of the biologically relevant information present in the images, resulting in predictions that are free of artefacts. This phenonemon has been observed in other applications such as optoacoustic image reconstruction from sparse data [37]. While the underlying reason is not yet understood, we speculate that the artefacts may not be occurring consistently enough to provide a strong signal compared to the signal of interest. Unless the model is overfitted, it may then not learn to reproduce the z-projection images completely according to the training data and may hence not include these artefacts. To investigate the reason for how CNN-based image reconstruction may provide implicit artefact removal is something we leave for future research.

Although we compare two different CNN-architectures, specifically showing that a modified U-Net and using OSA-blocks is preferable to a standard U-Net generator, we do no extensive study of how architectural elements affect the result. CNN-architecture design is a large field of study and there are vast permutations and options for how to design the CNN optimally. While we chose OSA-blocks, we could have obtained similar results by choosing other architectural variants that have demonstrated better performance than the straightforward fully convolutional blocks used in the standard U-Net (e.g., split-attention blocks [38] or dense blocks [39]). Similarly, many variants of generative adversarial networks have been described in recent literature. We chose the 
conditional GAN variant due to its extensive track record of successful applications, including remote sensing image analysis [40], stain-to-stain-translation in histopathological images [41], translating between different type of magnetic resonance imaging-weighting [42], and more. The main focus of this study is to demonstrate the combination of axial z-sweep acquisition and deep learning-based image restoration and we leave an extensive evaluation of $\mathrm{CNN}$-architectures and training methodologies for future research.

Our deep learning models were solely trained on single-plane multi-spheroid data and managed to generated good results on data from all three experimental settings, exhibiting model transferability across plates. We note that the PSNR and SSIM score when evaluating on single-spheroid data was better for the input Z-sweep images than the deep-learning model projections (Table. 1). The FID metrics on the other hand showed a clear improvement for the projections which is in line with can be visibly be observed (Fig. 6.a). Interestingly, we see in the single-spheroid model that the PSNR and the SSIM are actually higher in the z-sweep images than the others. However, there are certain caveats related to the z-sweep images that are important to consider in relation to these metrics. Because the z-sweep has a much longer effective exposure time than the z-stack projections, z-sweeps exhibit less shot noise. However, because the z-sweep acquisition moves through the sample so quickly, it also captures less photons at a given z-location resulting in the overall signal being lower. Applying this to our different biological models, the large, very bright single spheroid model, metrics are likely less affected by the signal difference in the acquisition and more impacted by shot noise. Whereas in the multi-spheroid models, the smaller, dimmer spheroids bias the metrics toward changes in signal. Because of these caveats, we feel that the FID score, which is affected by noise and blurriness but less so overall intensity, is the most meaningful the the metrics investigated.

While our results detail an important first step to enable this workflow for high-throughput fluorescence imaging, there are certain limitations. Here, we have tested three common different experimental preparations for culturing tumor spheroids which range from a single, relatively large spheroid to hundreds of spheroids embedded throughout a volume of extracellular matrix (Fig. 4). While this demonstrates the applicability of our proposed method to a variety of 3D samples, the world of 3D 
imaging in biology is incredibly diverse. Future investigations will certainly focus on

\section{Conclusions}

Widefield fluorescence microscopy is commonly used to study biological phenomena. However, fluorescent microscopic imaging of complex 3D samples, such as tumor spheroids, is burdensome since it often entails the collection of a stack of multiple optical sections along the z-dimension, which is time-consuming and has high risk of causing phototoxicity. To alleviate this problem, we propose a workflow combining axial z-sweep acquisition and deep learning-based image enhancement. Axial z-sweep acquisition is many times faster than the corresponding z-stack acquisition but suffer from poor image quality. Using deep convolutional neural networks and conditional generative adversarial networks, we train models to predict high quality projection images of z-stacks based on z-sweep images. We demonstrate that our proposed model, OSA-CGAN, outperforms baseline models in terms of visual quality, quantitatively and qualitatively, in three case studies of tumor spheroid imaging. OSA-CGAN is able to produce perceptually sharp images without introducing systematic bias in fluorescent imaging of single-, multi- and embedded tumor spheroids. To conclude, this work shows how axial z-sweep acquisition and deep learning enable high-throughput and high-quality widefield fluorescence microscopy imaging of complex 3D samples.

\section{References}

1. Magidson V, Khodjakov A. Circumventing photodamage in live-cell microscopy. Methods in Cell Biology. 2013;114:545-560. 
2. Elliott AD. Confocal microscopy: principles and modern practices. Current protocols in cytometry. 2020;92(1):e68.

3. Bayguinov PO, Oakley DM, Shih CC, Geanon DJ, Joens MS, Fitzpatrick JA. Modern laser scanning confocal microscopy. Current protocols in cytometry. 2018;85(1):e39.

4. Chang BJ, Manton JD, Sapoznik E, Pohlkamp T, Terrones TS, Welf ES, et al. Real-time multi-angle projection imaging of biological dynamics. Nature Methods. 2021;18(7):829-834. doi:10.1038/s41592-021-01175-7.

5. Carpenter AE, Jones TR, Lamprecht MR, Clarke C, Kang IH, Friman O, et al. CellProfiler: image analysis software for identifying and quantifying cell phenotypes. Genome biology. 2006;7(10):1-11.

6. Schindelin J, Arganda-Carreras I, Frise E, Kaynig V, Longair M, Pietzsch T, et al. Fiji: an open-source platform for biological-image analysis. Nature methods. $2012 ; 9(7): 676-682$.

7. Duval K, Grover H, Han LH, Mou Y, Pegoraro AF, Fredberg J, et al. Modeling Physiological Events in 2D vs. 3D Cell Culture. Physiology. 2017;32(4):266-277. doi:10.1152/physiol.00036.2016.

8. Kapalczyńska M, Kolenda T, Przybyla W, Zajaczkowska M, Teresiak A, Filas V, et al. 2D and 3D cell cultures - a comparison of different types of cancer cell cultures. Archives of Medical Science. 2018;14(4):910-919. doi:10.5114/aoms.2016.63743.

9. Chen BC, Legant WR, Wang K, Shao L, Milkie DE, Davidson MW, et al. Lattice light-sheet microscopy: Imaging molecules to embryos at high spatiotemporal resolution. Science. 2014;346(6208). doi:10.1126/science.1257998.

10. Woringer M, Darzacq X, Zimmer C, Mir M. Faster and less phototoxic 3D fluorescence microscopy using a versatile compressed sensing scheme. Optics express. 2017;25(12):13668-13683.

11. Joon Ho D, Fu C, Salama P, Dunn KW, Delp EJ. Nuclei segmentation of fluorescence microscopy images using three dimensional convolutional neural 
networks. In: Proceedings of the IEEE Conference on Computer Vision and Pattern Recognition Workshops; 2017. p. 82-90.

12. Ho DJ, Fu C, Salama P, Dunn KW, Delp EJ. Nuclei detection and segmentation of fluorescence microscopy images using three dimensional convolutional neural networks. In: 2018 IEEE 15th International Symposium on Biomedical Imaging (ISBI 2018). IEEE; 2018. p. 418-422.

13. Stegmaier J, Spina TV, Falcao AX, Bartschat A, Mikut R, Meyerowitz E, et al. Cell segmentation in 3D confocal images using supervoxel merge-forests with CNN-based hypothesis selection. In: 2018 IEEE 15th International Symposium on Biomedical Imaging (ISBI 2018). IEEE; 2018. p. 382-386.

14. Castelli FM, Roffilli M, Mazzamuto G, Costantini I, Silvestri L, Pavone FS. Semantic Segmentation of Neuronal Bodies in Fluorescence Microscopy Using a 2D+3D CNN Training Strategy with Sparsely Annotated Data. In: International Conference on Machine Learning, Optimization, and Data Science. Springer; 2020. p. $95-99$.

15. Weigert M, Royer L, Jug F, Myers G. Isotropic reconstruction of 3D fluorescence microscopy images using convolutional neural networks. In: International Conference on Medical Image Computing and Computer-Assisted Intervention. Springer; 2017. p. 126-134.

16. Weigert M, Schmidt U, Boothe T, Müller A, Dibrov A, Jain A, et al. Content-aware image restoration: pushing the limits of fluorescence microscopy. Nature Methods. 2018;15(12):1090-1097.

17. Zhang H, Fang C, Xie X, Yang Y, Mei W, Jin D, et al. High-throughput, high-resolution deep learning microscopy based on registration-free generative adversarial network. Biomed Opt Express. 2019;10(3):1044-1063. doi:10.1364/BOE.10.001044.

18. Wu Y, Rivenson Y, Wang H, Luo Y, Ben-David E, Bentolila LA, et al. Three-dimensional virtual refocusing of fluorescence microscopy images using deep learning. Nature methods. 2019;16(12):1323-1331. 
19. Wu Y, Rivenson Y, Wang H, Luo Y, Ben-David E, Bentolila LA, et al. Deep-Z: 3D Virtual Refocusing of Fluorescence Images Using Deep Learning. In: CLEO: Applications and Technology. Optical Society of America; 2020. p. AW3T-6.

20. Nehme E, Freedman D, Gordon R, Ferdman B, Weiss LE, Alalouf O, et al. DeepSTORM3D: dense 3D localization microscopy and PSF design by deep learning. Nature Methods. 2020;17(7):734-740.

21. Page J, Favaros P. Learning to Model and Calibrate Optics Via a Differentiable Wave Optics Simulator. In: 2020 IEEE International Conference on Image Processing (ICIP). IEEE; 2020. p. 2995-2999.

22. Lim S, Ye JC. Blind deconvolution microscopy using cycle consistent CNN with explicit PSF layer. In: International Workshop on Machine Learning for Medical Image Reconstruction. Springer; 2019. p. 173-180.

23. Lee S, Han S, Salama P, Dunn KW, Delp EJ. Three dimensional blind image deconvolution for fluorescence microscopy using generative adversarial networks. In: 2019 IEEE 16th International Symposium on Biomedical Imaging (ISBI 2019). IEEE; 2019. p. 538-542.

24. Huang L, Chen H, Luo Y, Rivenson Y, Ozcan A. Recurrent neural network-based volumetric fluorescence microscopy. Light: Science \& Applications. 2021;10(1):1-16.

25. Ronneberger O, Fischer P, Brox T. U-net: Convolutional networks for biomedical image segmentation. In: International Conference on Medical image computing and computer-assisted intervention. Springer; 2015. p. 234-241.

26. Lee Y, Park J. CenterMask: Real-Time Anchor-Free Instance Segmentation. In: 2020 IEEE/CVF Conference on Computer Vision and Pattern Recognition (CVPR); 2020. p. 13903-13912.

27. Hu J, Shen L, Sun G. Squeeze-and-excitation networks. In: Proceedings of the IEEE conference on computer vision and pattern recognition; 2018. p. 7132-7141. 
28. Isola P, Zhu JY, Zhou T, Efros AA. Image-to-Image Translation with Conditional Adversarial Networks. In: 2017 IEEE Conference on Computer Vision and Pattern Recognition (CVPR); 2017. p. 5967-5976.

29. Salimans T, Goodfellow I, Zaremba W, Cheung V, Radford A, Chen X, et al. Improved Techniques for Training GANs. In: Lee D, Sugiyama M, Luxburg U, Guyon I, Garnett R, editors. Advances in Neural Information Processing Systems. vol. 29. Curran Associates, Inc.; 2016.

30. Goodfellow IJ, Pouget-Abadie J, Mirza M, Xu B, Warde-Farley D, Ozair S, et al. Generative Adversarial Networks. arXiv:14062661 [cs, stat]. 2014;.

31. Costa EC, Moreira AF, de Melo-Diogo D, Gaspar VM, Carvalho MP, Correia IJ. 3D tumor spheroids: an overview on the tools and techniques used for their analysis. Biotechnology advances. 2016;34(8):1427-1441.

32. ; Image Processing and Segmentation of Sets of Z-Stacked Images of Three-Dimensional Biological Samples. US20210327080A1; April 21, 2020.

33. Forster B, Van De Ville D, Berent J, Sage D, Unser M. Extended depth-of-focus for multi-channel microscopy images: a complex wavelet approach. In: 2004 2nd IEEE International Symposium on Biomedical Imaging: Nano to Macro (IEEE Cat No. 04EX821). IEEE; 2004. p. 660-663.

34. Kingma DP, Ba JL. Adam: A method for stochastic gradient descent. In: ICLR: International Conference on Learning Representations; 2015. p. 1-15.

35. Forsgren E. ProjSweep; 2021.

https://github.com/edvinforsgren/ProjSweep.

36. Heusel M, Ramsauer H, Unterthiner T, Nessler B, Hochreiter S. GANs Trained by a Two Time-Scale Update Rule Converge to a Local Nash Equilibrium. In: Guyon I, Luxburg UV, Bengio S, Wallach H, Fergus R, Vishwanathan S, et al., editors. Advances in Neural Information Processing Systems. vol. 30. Curran Associates, Inc.; 2017.

37. Davoudi N, Deán-Ben XL, Razansky D. Deep learning optoacoustic tomography with sparse data. Nature Machine Intelligence. 2019;1(10):453-460. 
38. Zhang H, Wu C, Zhang Z, Zhu Y, Lin H, Zhang Z, et al. Resnest: Split-attention networks. arXiv preprint arXiv:200408955. 2020;.

39. Huang G, Liu Z, Van Der Maaten L, Weinberger KQ. Densely connected convolutional networks. In: Proceedings of the IEEE conference on computer vision and pattern recognition; 2017. p. 4700-4708.

40. Wang X, Yan H, Huo C, Yu J, Pant C. Enhancing Pix2Pix for remote sensing image classification. In: 2018 24th International Conference on Pattern Recognition (ICPR). IEEE; 2018. p. 2332-2336.

41. Salehi P, Chalechale A. Pix2pix-based stain-to-stain translation: A solution for robust stain normalization in histopathology images analysis. In: 2020 International Conference on Machine Vision and Image Processing (MVIP). IEEE; 2020. p. 1-7.

42. Mori M, Fujioka T, Katsuta L, Kikuchi Y, Oda G, Nakagawa T, et al. Feasibility of new fat suppression for breast MRI using pix2pix. Japanese Journal of Radiology. 2020;38(11):1075-1081. 
(a)

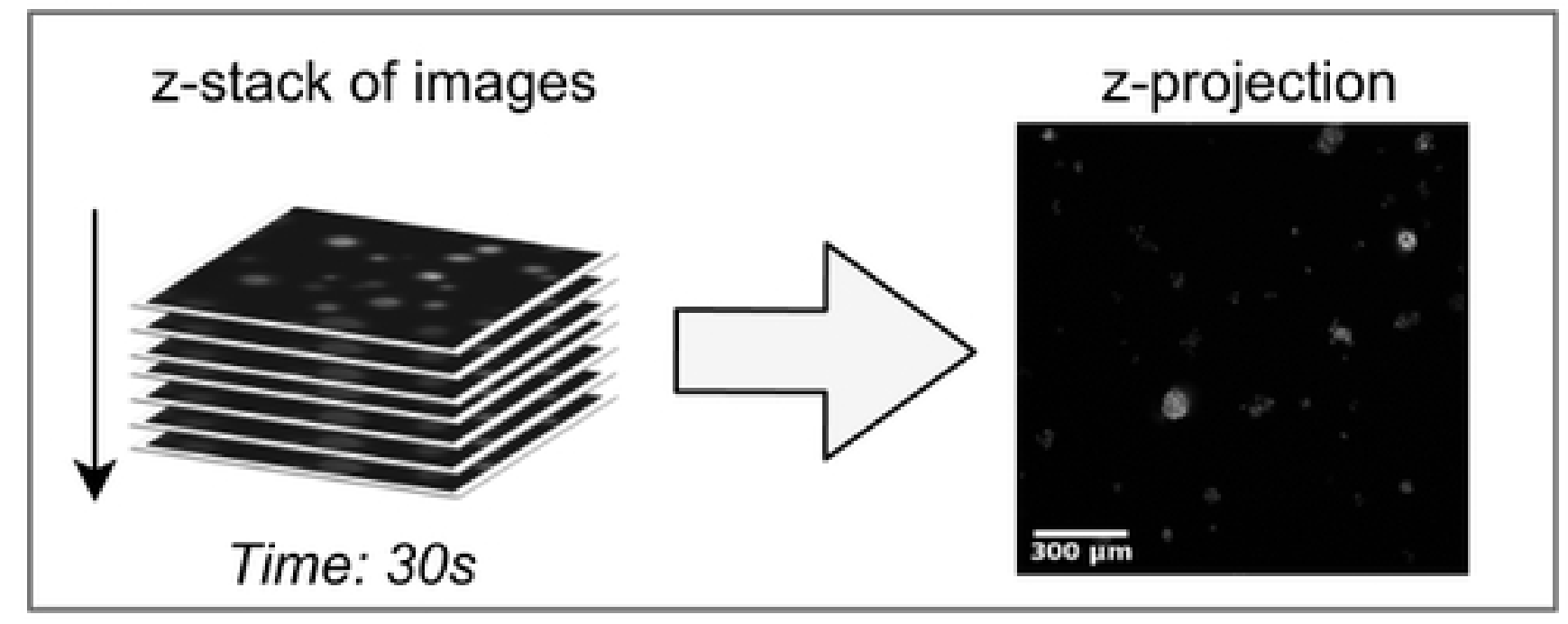

(b)

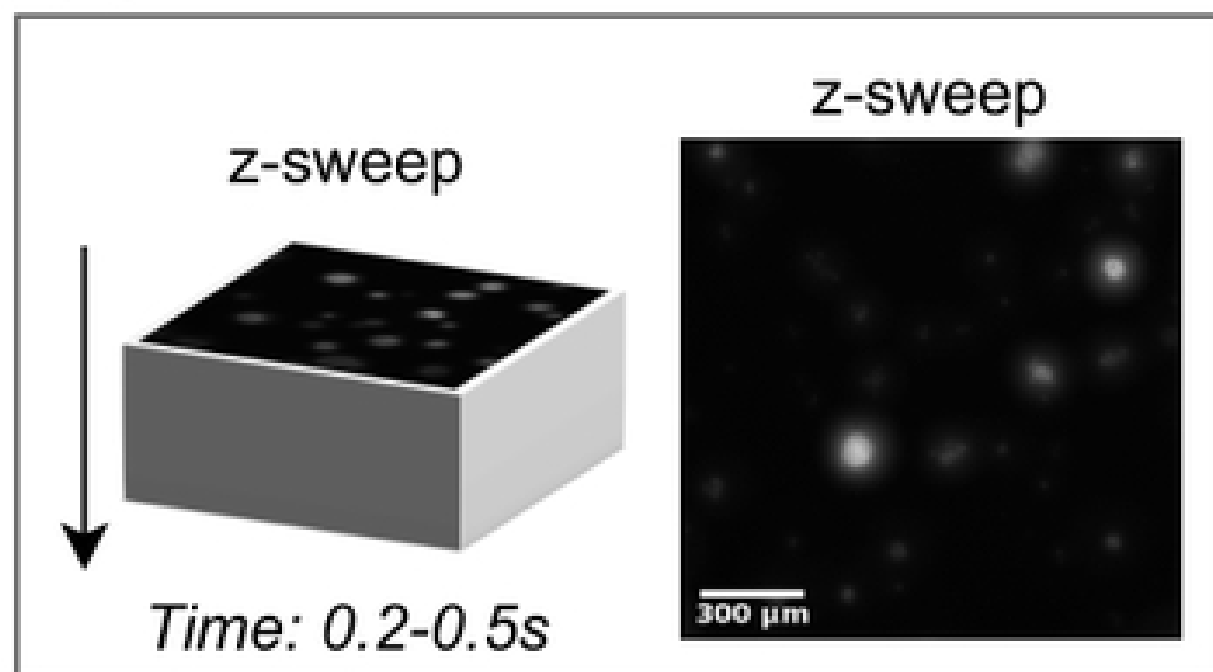

(c)

z-sweep

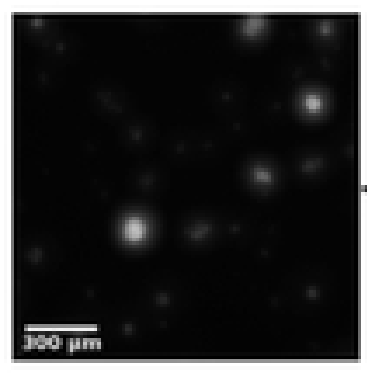

z-projection

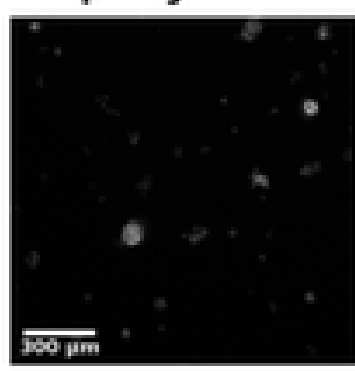

z-sweep

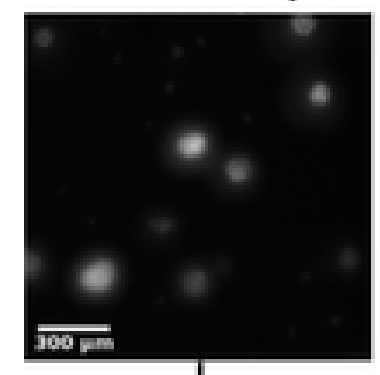

Trained model

\section{Predicted z-projection}

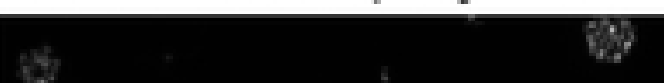

Q⿻ 


\section{(a) U-Net}

132

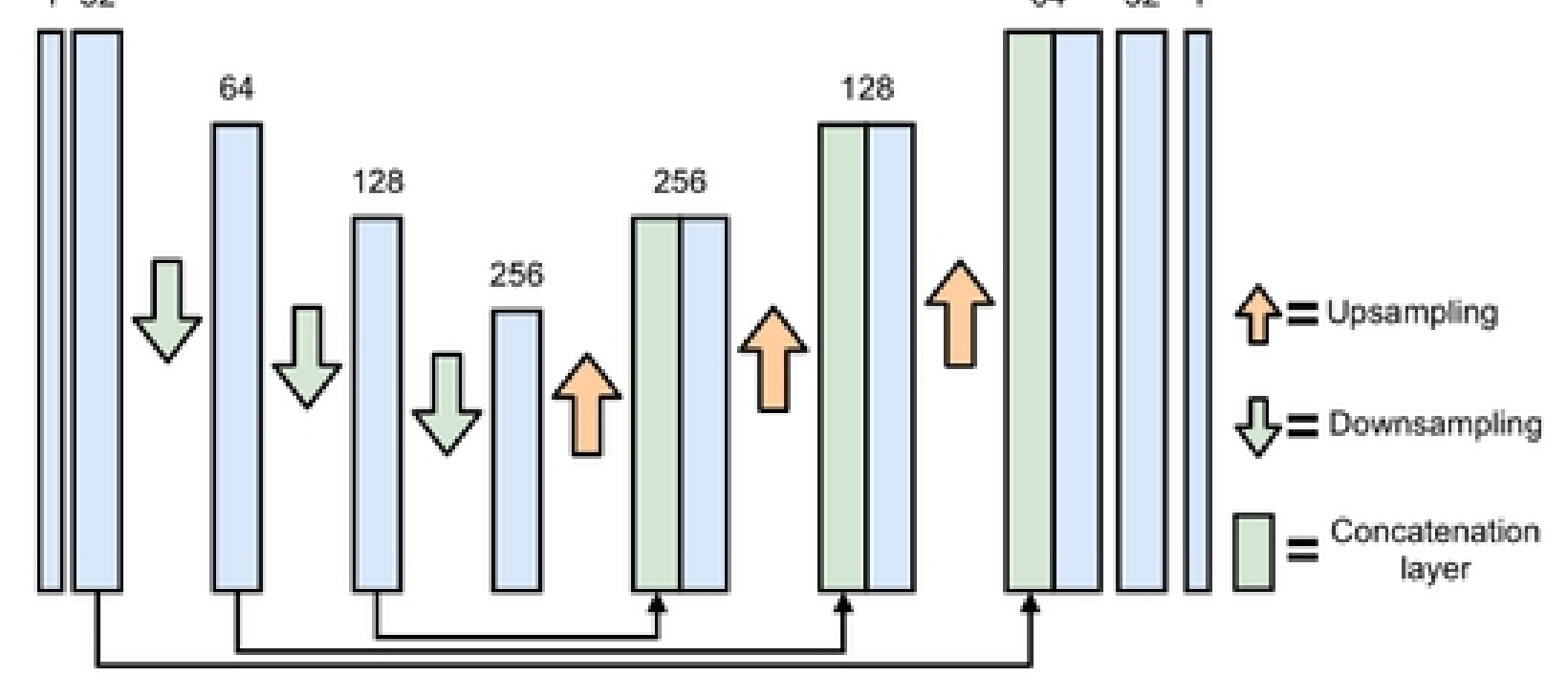

\section{$\frac{\text { (c) PatchGAN }}{2}$}

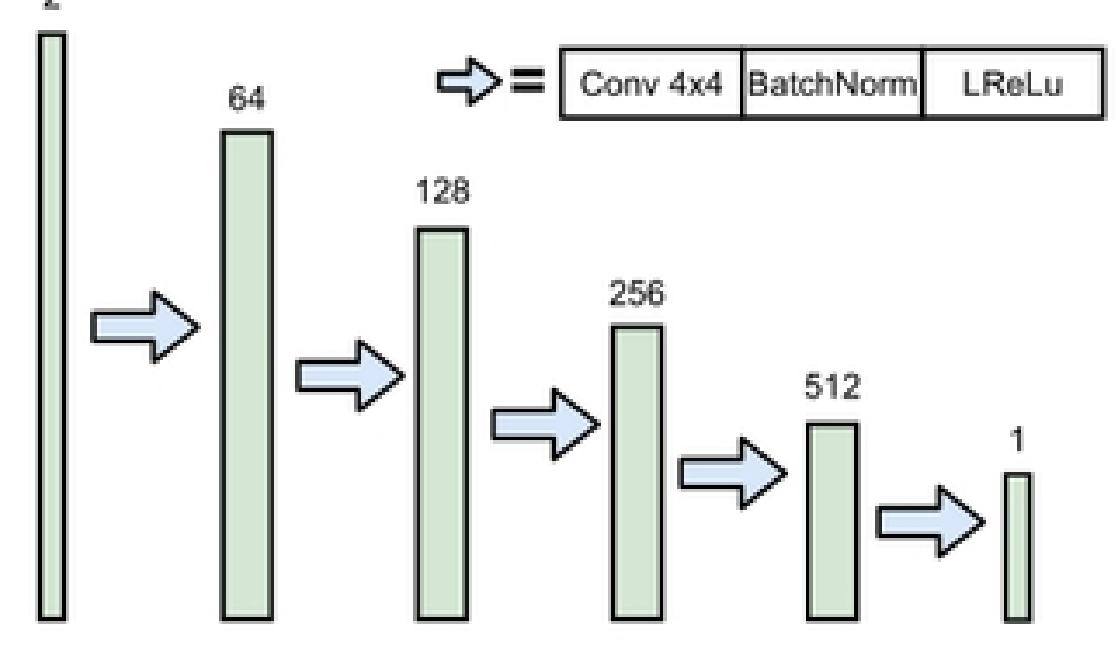

(b) Standard U-Net

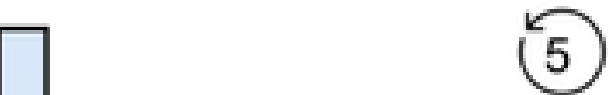

\begin{tabular}{|c|c|c|}
\hline$F_{3 \times 3}$ & BatchNorm & ReLu \\
\hline
\end{tabular}

(d) OSA-block

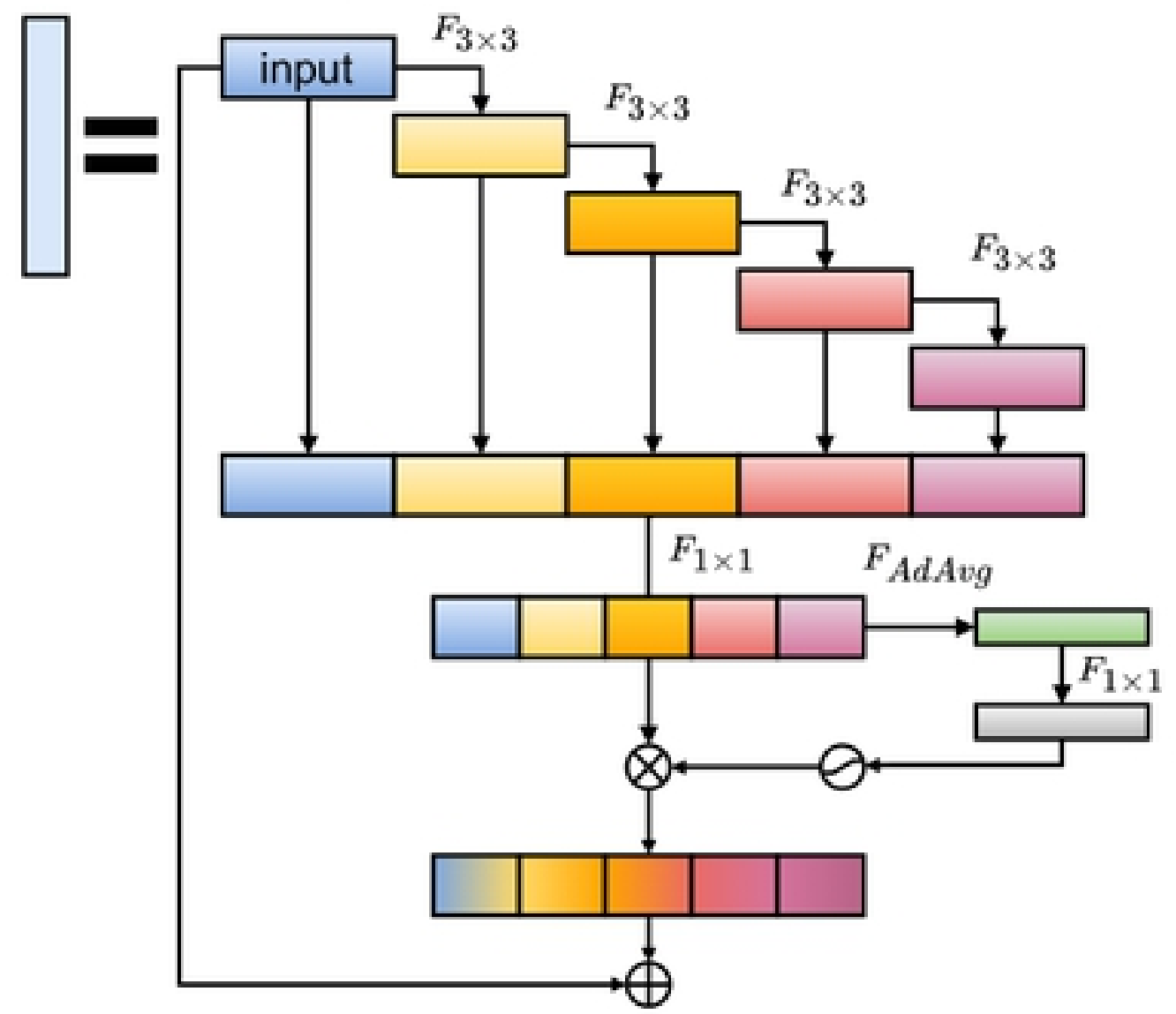




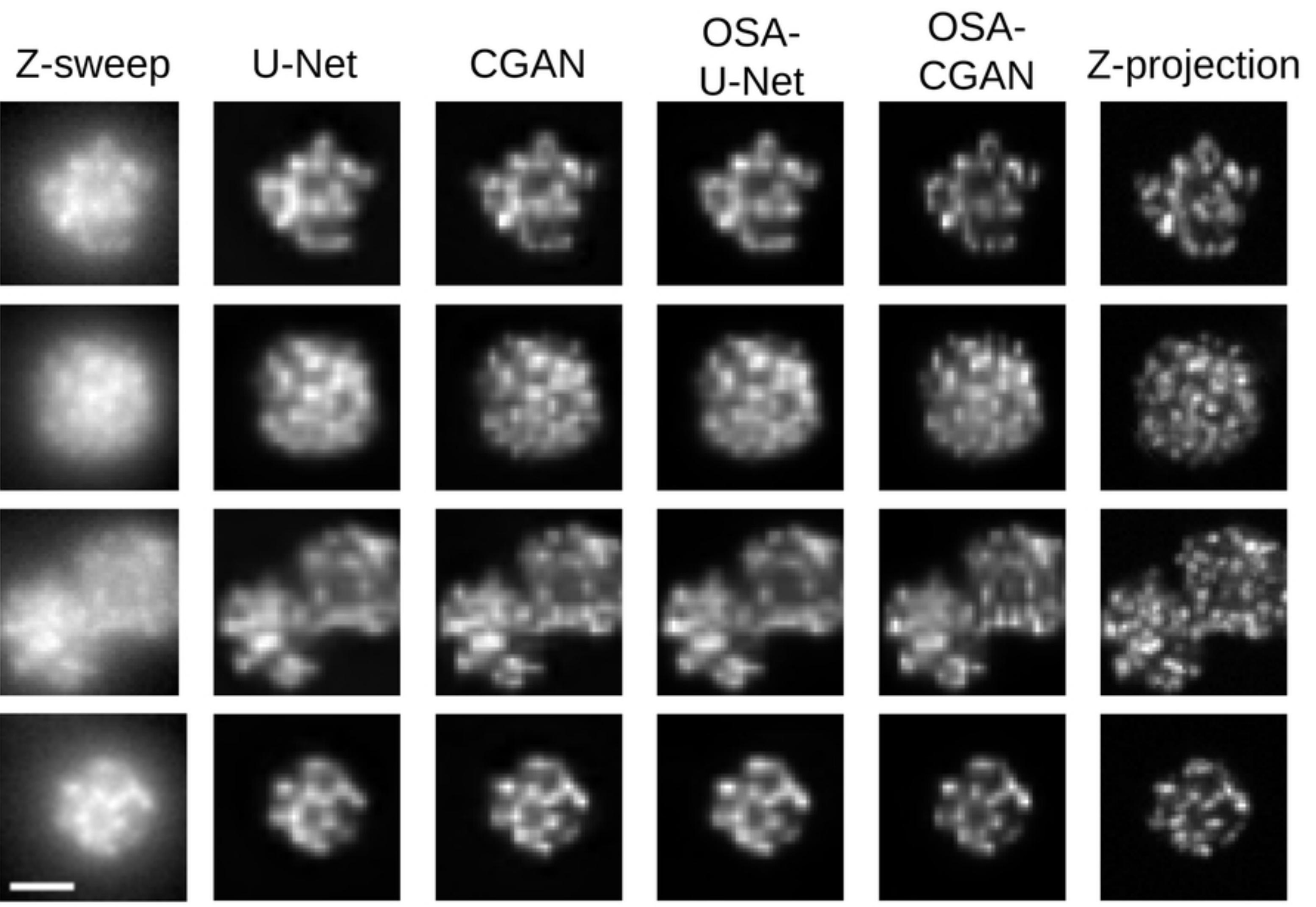


(a) Z-sweep

Prediction

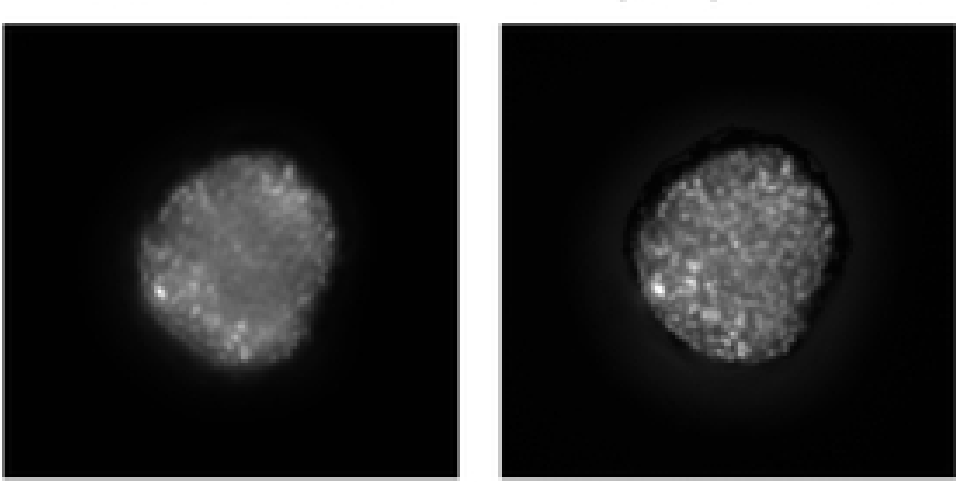

20.

$\overline{300 \mu \mathrm{m}}$
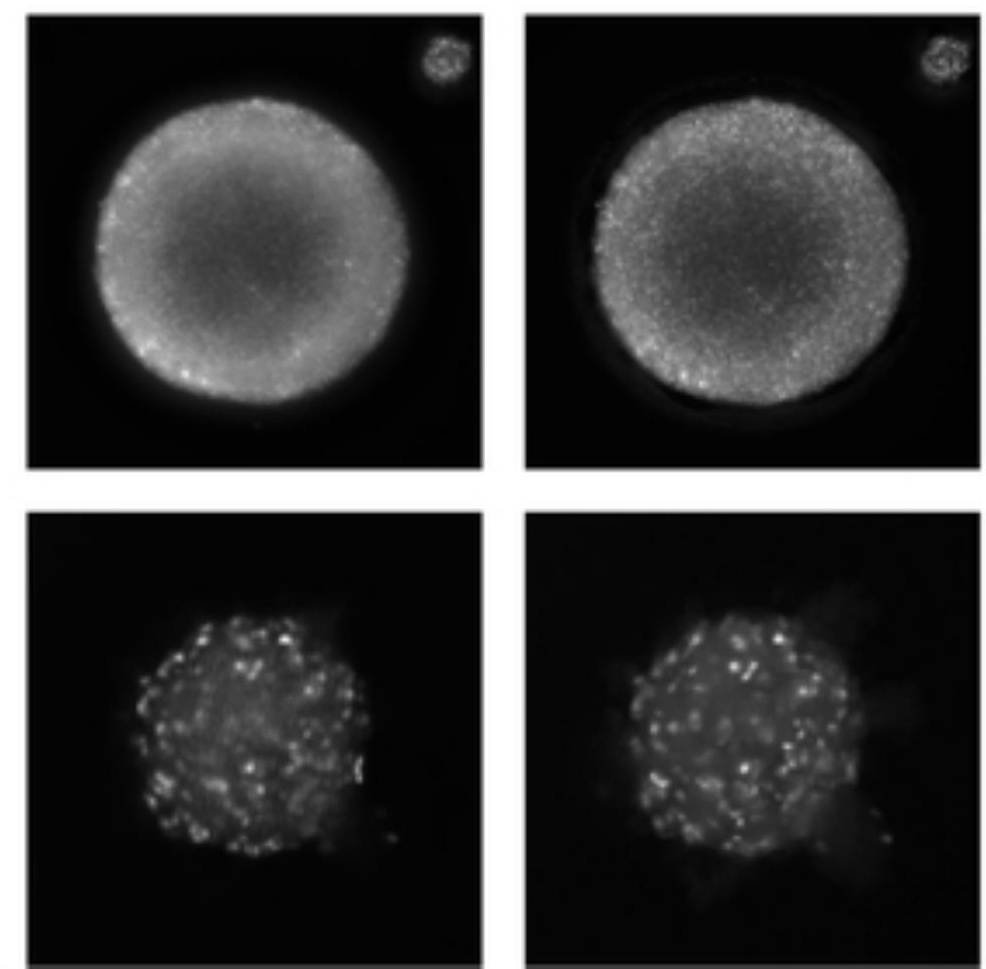

Z-projection

(b) Z-sweep
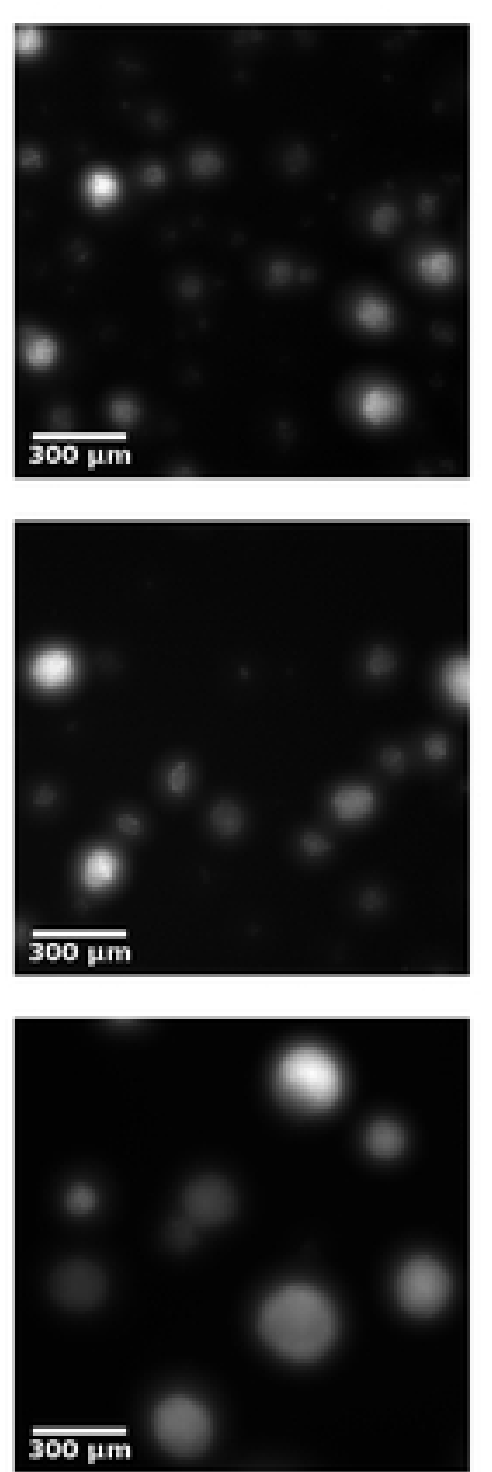

Prediction
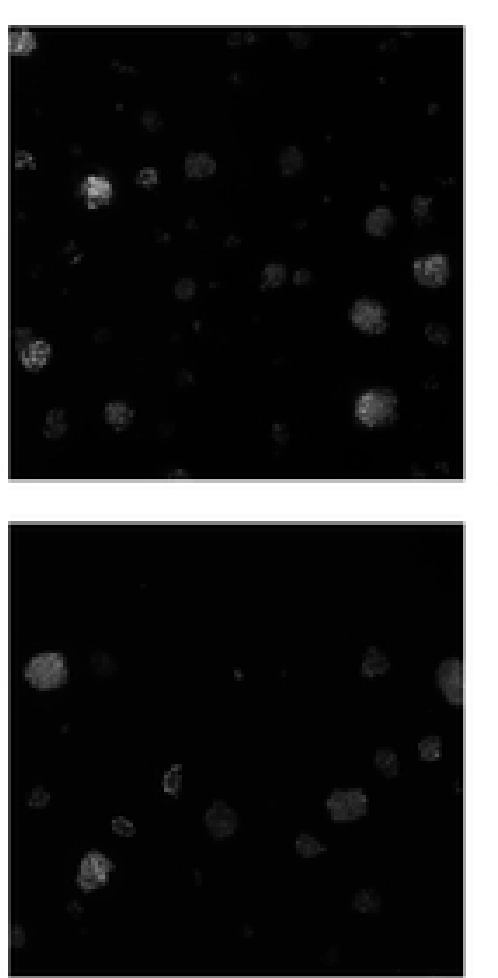

Z-projection
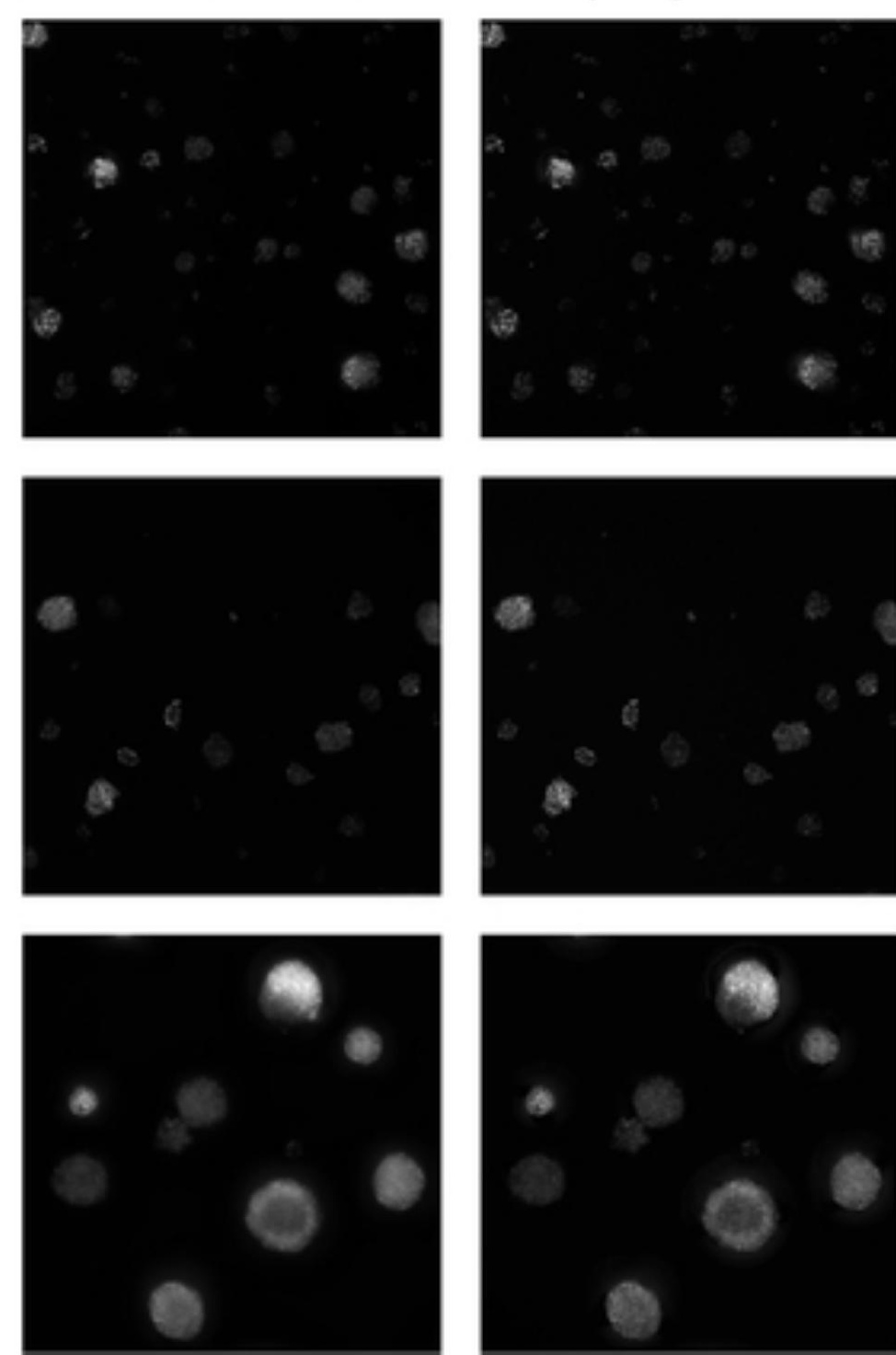

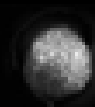

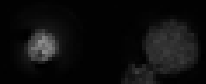
0

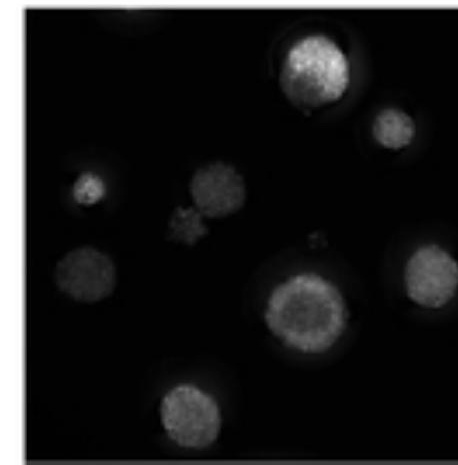


(a)

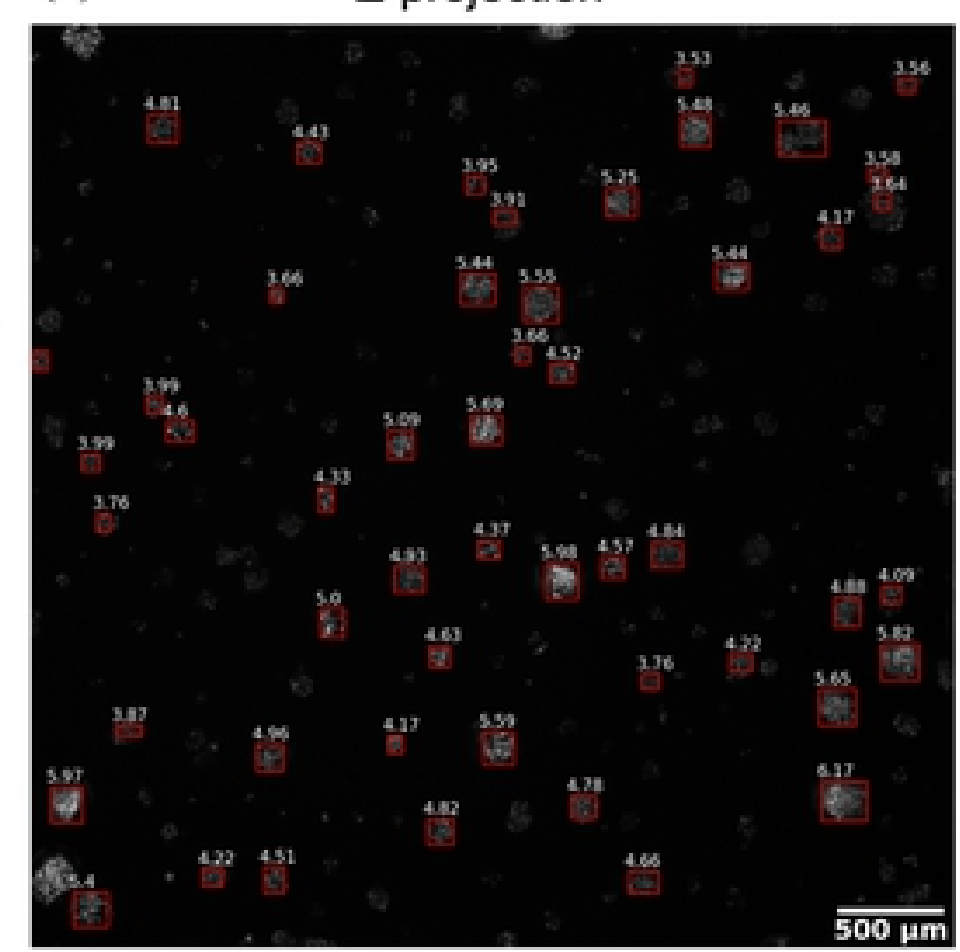

(b)

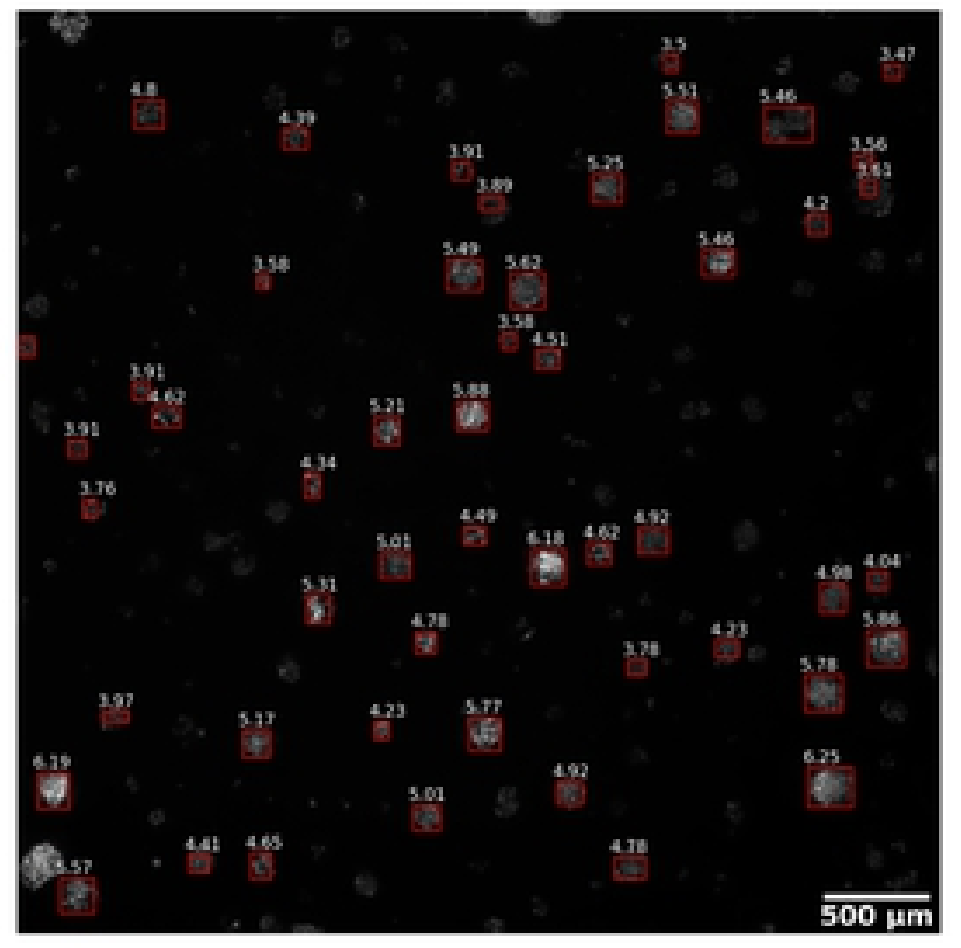

(c)

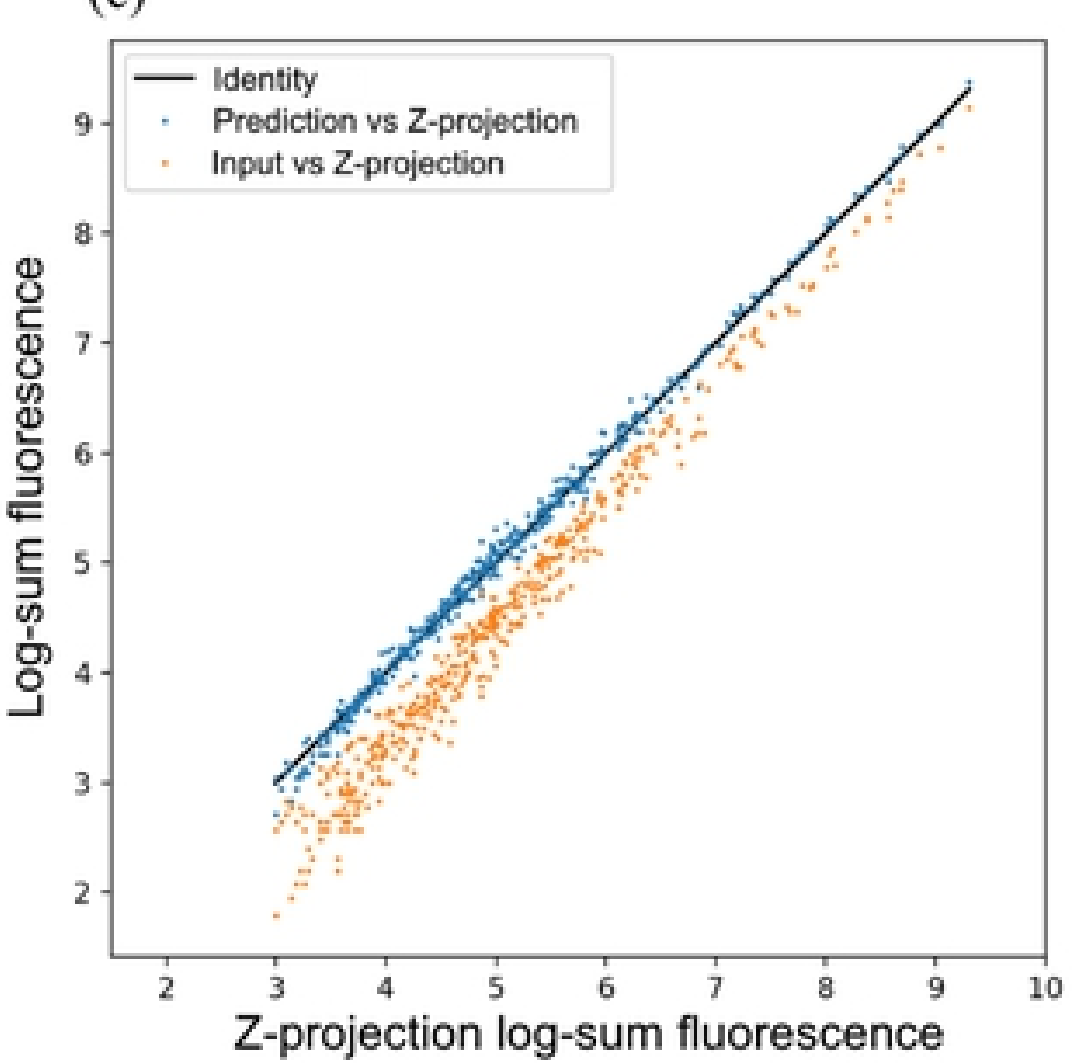


